\title{
Dabrafenib and its potential for the treatment of metastatic melanoma
}

\author{
This article was published in the following Dove Press journal: \\ Drug Design, Development and Therapy \\ 10 December 2012 \\ Number of times this article has been viewed
}

\author{
Alexander M Menzies' \\ Georgina V Long',2 \\ Rajmohan Murali 3,4 \\ 'Melanoma Institute Australia, \\ Sydney, New South Wales, Australia; \\ ${ }^{2}$ Westmead Institute for Cancer \\ Research and Crown Princess \\ Mary Cancer Centre Westmead, \\ Sydney, New South Wales, Australia; \\ ${ }^{3}$ Department of Pathology; ${ }^{4}$ Human \\ Oncology and Pathogensis Program, \\ Memorial Sloan-Kettering Cancer \\ Center, New York, NY, USA
}

\begin{abstract}
The purpose of this study is to review the development of BRAF inhibitors, with emphasis on the trials conducted with dabrafenib (GSK2118436) and the evolving role of dabrafenib in treatment for melanoma patients. Fifty percent of cutaneous melanomas have mutations in $B R A F$, resulting in elevated activity of the mitogen-activated protein kinase signaling pathway. Dabrafenib inhibits the mutant BRAF (BRAFmut) protein in melanomas with $B R A F^{V 600 E}$ and $B R A F^{V 600 K}$ genotypes. $B R A F^{V 600 E}$ metastatic melanoma patients who receive dabrafenib treatment exhibit high clinical response rates and compared with dacarbazine chemotherapy, progression-free survival. Efficacy has also been demonstrated in $B R A F^{V 600 K}$ patients and in those with brain metastases. Dabrafenib has a generally mild and manageable toxicity profile. Cutaneous squamous cell carcinomas and pyrexia are the most significant adverse effects. Dabrafenib appears similar to vemurafenib with regard to efficacy but it is associated with less toxicity. It is expected that new combinations of targeted drugs, such as the combination of dabrafenib and trametinib (GSK1120212, a MEK inhibitor), will provide higher response rates and more durable clinical benefit than dabrafenib monotherapy.
\end{abstract}

Keywords: BRAF mutation, BRAF inhibitor, clinical trial, GSK1120212, GSK2118436, vemurafenib

\section{Introduction}

Patients with advanced melanoma have a poor prognosis, with 1-year survival rates as low as $33 \%$ and a median overall survival (OS) of approximately 9 months. ${ }^{1}$ Until recently, systemic treatments were largely ineffective. Following the discovery of $B R A F$ mutations in melanoma, ${ }^{2}$ there has been rapid development of selective BRAF inhibitors, leading to a major shift in the approach to treating patients with BRAFmutated $\left(B R A F^{m u t}\right)$ melanoma. Dabrafenib is a selective BRAF inhibitor characterized by high response rates, a rapid mode of action, little toxicity, and a prolonged progression-free survival (PFS) over chemotherapy. ${ }^{3}$ The authors review the development of BRAF inhibitors, with emphasis on the trials conducted with dabrafenib and its evolving role in treating melanoma patients.

\section{Systemic therapy in the pre-BRAF era}

Prior to the development of selective BRAF inhibitors and newer immunotherapies, ${ }^{4,5}$ metastatic melanoma was largely refractory to systemic treatment. Dacarbazine (DTIC) was the most commonly used systemic agent. Trials with DTIC and other chemotherapeutic agents (alone or in combination with interferon or interleukin-2) demonstrated response rates below 20\%, a median PFS of less than 4 months, and OS 
of less than 9 months. $^{6-11}$ Interleukin-2 trials reported durable complete responses in less than $10 \%$ of patients, but at the cost of significant toxicity. ${ }^{12}$

\section{BRAF mutations in melanoma Discovery of BRAF mutations}

A decade ago, a seminal study investigating the mitogenactivated protein kinase (MAPK) pathway identified mutations in the activating segment of the $B R A F$ kinase domain. ${ }^{2}$ Ninety percent of the mutations in $B R A F$ involved exon $15(1799 \mathrm{~T}>\mathrm{A})$, resulting in the substitution of valine by glutamic acid at residue 600 (initially thought to be 599); this mutation was designated $B R A F^{V 600 E}$. Sixty percent of the melanomas and cell lines investigated in this study harbored the $B R A F^{V 600 E}$ mutation. ${ }^{2}$ The MAPK pathway is a major intracellular signal transduction pathway that is responsible for cellular proliferation, gene expression, differentiation, mitosis, cell survival, and apoptosis (Figure 1). Melanoma cell lines with $B R A F$ mutations had elevated kinase activity compared with those with wild-type $B R A F$ $\left(B R A F^{w t}\right)$, and they signaled through the MAPK pathway without upstream activation by RAS, indicating that the cells lost their dependence on upstream signaling. ${ }^{2}$ Furthermore, when $B R A F^{V 600 E}$ was ectopically expressed in fibroblast cell lines, it caused hyperstimulation of the MAPK cascade and malignant transformation. ${ }^{2}$ Subsequent validation studies of melanoma cell lines identified frequent activating mutations in codon 600 of $B R A F .^{13}$

\section{Effects of mutated BRAF in melanoma}

In vitro data indicate that mutant $B R A F$ is oncogenic. ${ }^{2,14}$ $B R A F$ mutations are very common in melanocytic nevi ${ }^{15}$ and are less prevalent in primary ${ }^{16-21}$ and metastatic ${ }^{22-24}$ melanoma. This suggests that the $B R A F$ mutation is an early event in neoplastic transformation of melanocytes but is insufficient in itself to lead to malignancy. ${ }^{25}$ Indeed, the formation of melanocytic nevi appears to result from transformation of melanocytes by $B R A F$ mutation, proliferation, and then oncogene $(B R A F)$-induced senescence. Progression from nevus to melanoma requires escape from senescence. ${ }^{26-28}$ The majority of melanomas are thought to arise de novo from transformed melanocytes without progression through a nevus; in such cases, other genetic alterations (eg, alterations in the $\mathrm{p} 53$ and $\mathrm{Rb}$ pathways) cooperate with oncogenic $B R A F$ mutations to drive malignant transformation. ${ }^{29}$ Further research is required to fully understand the interplay between $B R A F$ mutations and other factors in melanoma development. Furthermore, the role of mutant $B R A F$ in melanoma progression from primary to metastatic disease is not clear. Several retrospective studies of stage III

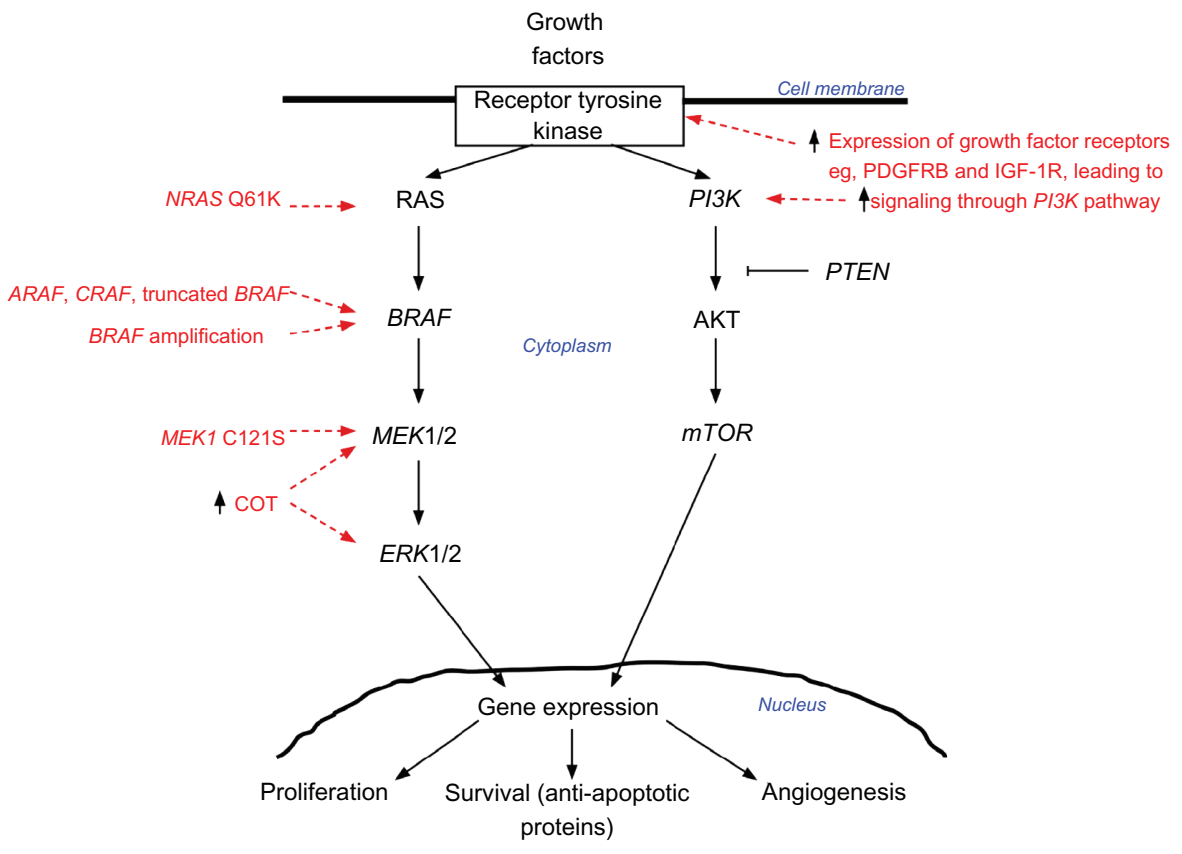

Figure I Simplified scheme of the mitogen-activated protein kinase (MAPK) (RAS-RAF-MEK-ERK) signaling pathway.

Notes: BRAF and NRAS are the most commonly mutated oncogenes in melanoma. Downstream effectors of the pathway lead to increased survival and proliferation by various mechanisms (eg, increased transcription of genes and regulation of apoptotic proteins). Potential mechanisms of acquired resistance to BRAF inhibitor therapy are highlighted in red. BRAF inhibition can lead to activation of the MAPK pathway via acquisition of secondary NRAS Q6IK mutations; RAF isoform signal switching (ARAF > BRAF > CRAF); truncation of $B R A F$, resulting in a form that is resistant to the inhibitor; amplification of BRAF; MEKI CI2IS mutation; and increased expression of COT, a partner kinase. Abbreviations: PDGFRB, platelet-derived growth factor receptor beta; IGF-IR, insulin-like growth factor I receptor. 
and IV melanoma patients report conflicting results with regard to the associations of $B R A F$ mutation status with distant metastasis-free survival and OS..$^{22,23,30-33}$ However, studies with consecutive accrual ${ }^{22,23,33}$ report poorer survival in $B R A F^{m u t}$ patients than in $B R A F^{w t}$ patients.

\section{BRAF mutation prevalence, genotypes, and clinicopathologic correlates}

$B R A F$ mutations occur in approximately $50 \%$ of cutaneous melanomas ${ }^{22-24,34}$ and most commonly occur in exon 15 , at codon 600 (V600). ${ }^{35}$ Over $75 \%$ of BRAF mutations are characterized by the substitution of valine by glutamic acid at residue 600 (V600E). ${ }^{22-24} \mathrm{~A}$ less common genotype is $\mathrm{V} 600 \mathrm{~K}$, a substitution of valine by lysine. The prevalence of $\mathrm{V} 600 \mathrm{~K}$ varies by geographic region, ranging from less than $10 \%$ in northern Europe ${ }^{18,24,36}$ to $20 \%-30 \%$ in Australia, Texas, and Florida, ${ }^{23,34,37,38}$ likely reflecting differences in ambient ultraviolet (UV) exposure. ${ }^{34}$ Rare V600 mutations include V600R and V600D. The frequency of $B R A F$ mutations in melanoma is inversely proportional to patient age,,$^{34,39}$ and there are reported associations of $B R A F$ mutation status and genotype with anatomic location of primary melanoma, histological subtype, and evidence of cumulative sun damage. ${ }^{22,39}$ Acral and mucosal melanomas have a lower frequency of $B R A F$ mutations, ${ }^{16,33,40}$ and uveal melanomas do not harbor $B R A F$ mutations. ${ }^{41-43}$

\section{Development of BRAF inhibitors}

Since the identification of $B R A F$ mutations in cutaneous melanomas, much effort has been directed toward the development of pharmacologic agents that inhibit the mutated BRAF protein for treatment of melanoma patients. Two types of BRAF inhibitors have been developed and tested in advanced melanoma: type 1 BRAF inhibitors that bind and inhibit the active conformation of the BRAF kinase, and type 2 BRAF inhibitors that bind to the inactive conformation of the kinase (Table 1). ${ }^{44}$

\section{Nonselective (type 2) BRAF inhibitors}

Initial attempts to target $\mathrm{BRAF}^{\text {mut }}$ protein with type 2 inhibitors failed to show clinical efficacy. This was thought to be due to nonselective kinase inhibition, which resulted in significant toxicity at doses well below those required to inhibit the MAPK pathway.

\section{Sorafenib}

Sorafenib was developed and approved for treatment of kidney and liver cancer prior to the identification of
Table I Summary of BRAF inhibitors in development

\begin{tabular}{ll}
\hline Drug & Clinical trials $^{\mathbf{a}}$ \\
\hline $\begin{array}{l}\text { RAF inhibitors, type I } \\
\text { Vemurafenib (PLX4032) } \\
\text { (derived from PLX4072) }\end{array}$ & Phase I, II, and III trials complete ${ }^{4,60,61}$ \\
$\begin{array}{l}\text { Dabrafenib } \\
\text { (GSK2 I I8436) }\end{array}$ & Phase I, II, and III trials complete ${ }^{3,54,69}$ \\
LGX8I8 & Phase I trial recruiting (NCT0I436656) \\
GDC0879 & Preclinical studies only, ${ }^{118}$ \\
RAF inhibitors, type not reported \\
ARQ736 & Po further clinical development planned \\
PF04880594 & Preclinical studies only, ${ }^{119}$ no further \\
AZ628 & clinical development planned \\
RAF inhibitors, type 2 & Preclinical studies only ${ }^{97}$ \\
XL28I/BMS908662 & Phase I trial complete \\
RAF265 & Phase I trial complete \\
Sorafenib & Phase I, II, and III trials complete \\
\hline
\end{tabular}

Notes: a Only single-agent clinical trials are shown - clinical trials of RAF inhibitors (vemurafenib, dabrafenib, BMS908662, RAF265, and sorafenib) combined with other systemic therapies are complete or under way; bphase I and III clinical trials of singleagent sorafenib were conducted in nonmelanoma solid tumors.

Abbreviation: NR, not reported.

$B R A F$ mutations in melanoma. Sorafenib binds to CRAF, $\mathrm{BRAF}^{\mathrm{wt}}$, and $\mathrm{BRAF}^{\mathrm{mut}}$ proteins, with little selectivity for one isoform over another. It also inhibits other kinases including vascular endothelial growth factor receptor 2 (VEGFR2), vascular endothelial growth factor receptor 3, platelet-derived growth factor, p38 MAPK, FLT, cKIT, FMA, and RET. ${ }^{45}$ Phase II clinical trials in metastatic melanoma patients failed to demonstrate any efficacy of sorafenib as monotherapy, and pharmacodynamic analyses suggested that only partial inhibition of BRAF signaling was achieved at the maximum tolerated dose (MTD). ${ }^{46,47}$ Despite this, a phase I/II trial in which sorafenib was given in combination with carboplatin and paclitaxel (CP) reported a high response rate and longer PFS than with standard chemotherapy in metastatic melanoma patients. ${ }^{48}$ Two phase III randomized trials comparing the combination of $\mathrm{CP}$ and sorafenib with the combination of $\mathrm{CP}$ and placebo failed to show a benefit for the $\mathrm{CP}$ and sorafenib arm. ${ }^{6,7}$ To date, no studies have detected any correlation between BRAF genotype (mutant versus wild-type) and response to sorafenib, either as a single agent or in combination with chemotherapy. ${ }^{48,49}$

\section{RAF265}

RAF265 is another small molecule inhibitor of multiple kinases including BRAF (mutant and wild-type), CRAF, VEGFR2, and platelet-derived growth factor receptors, 
with greatest potency against $\mathrm{BRAF}^{\mathrm{V} 600 \mathrm{E}}$ and VEGFR2. Unlike sorafenib, RAF265 is a more potent inhibitor of BRAF $^{\text {mut }}$ than BRAF ${ }^{\mathrm{wt}} .{ }^{50}$ Xenograft studies reported impressive responses in both $B R A F^{w t}$ and $B R A F^{m u t}$ tumors, ${ }^{51}$ but a phase I trial in $B R A F^{m u t}$ and $B R A F^{w t}$ metastatic melanoma patients was disappointing, with response rates of $16 \%$ and $13 \%$, respectively. ${ }^{52}$ Further trials of RAF265 monotherapy are not expected.

\section{Selective (type I) BRAF inhibitors}

The most promising results in patients with $B R A F^{m u t}$ melanoma have been seen with drugs designed to selectively target the mutated and activated form of the BRAF kinase. Type 1 inhibitors bind the active conformation of the kinase, inhibiting BRAF kinase and MAPK activity at doses well below the MTD. ${ }^{53,54}$ The three drugs in clinical use or undergoing investigation in human clinical trials are (1) vemurafenib, (2) dabrafenib (discussed later in this review), and LGX818 (Table 1). These inhibitors are associated with specific toxicities such as cutaneous squamous cell carcinoma (cuSCC) $)^{55}$ and with the rapid development of resistance.

\section{Vemurafenib}

The first selective BRAF inhibitor to enter clinical trials was vemurafenib (also designated PLX4032, PLX4720, and RG7204). Vemurafenib was developed alongside a companion real-time polymerase chain reaction-based assay ( cobas $^{\circledR} 4800$ BRAF V600 Mutation Test; Roche Molecular Diagnostics, Inc, Pleasanton, CA) designed to detect the $B R A F^{V 600 E}$ mutation. This test is highly sensitive and specific for the V600E $B R A F$ mutation but is less so for other V600 BRAF mutations (eg, V600K, V600R, V600D, or the 1799_1800TG > AA V600E mutation)..${ }^{56-59}$

Preclinical evidence demonstrated vemurafenib activity in $B R A F^{V 600 E}$ melanoma but not in $B R A F^{w t}$ melanoma. Clinical trials began in patients with solid tumors and then focused on those with cobas 4800-positive metastatic melanoma, demonstrating high activity, a rapid mode of action, and significant clinical benefit. ${ }^{4,60}$ Initial results from the phase III trial were reported in 2011, ${ }^{61}$ and recently more mature data has been presented. ${ }^{62}$ When used as a first-line agent in $B R A F^{V 600 E}$ metastatic melanoma, vemurafenib had a Response Evaluation Criteria in Solid Tumors (RECIST) response rate ${ }^{63}$ of $53 \%$, a median PFS of 6.9 months, and a median OS of 13.6 months, compared with conventional DTIC chemotherapy's response rate of $8 \%$, median PFS of 1.6 months, and median OS of 10 months. ${ }^{61,62}$ A phase I study in patients with brain metastases showed intracranial activity, ${ }^{64}$ and a phase II study is currently under way (NCT01378975). In phase II and III trials, vemurafenib was active in a small number of patients retrospectively identified with $B R A F^{V 600 K}$ tumors $(\mathrm{n}=20,4 \%$ of the total study population). ${ }^{4,60}$ The response rate was less than that in patients with $B R A F^{V 600 E}$ tumors, and no survival data were presented regarding these patients. There is anecdotal evidence for vemurafenib activity in tumors with other $B R A F$ mutations (including $B R A F^{V 600 R}$ and $B R A F^{V 600 D}$ ), and a trial to formally investigate activity in non-V600E $B R A F^{m u t}$ melanoma is under way (NCT01586195).

Vemurafenib has received worldwide regulatory approval for treatment of patients with V600 BRAF $F^{m u t}$ metastatic melanoma, although in the United States and Brazil the approval is restricted to patients with $B R A F^{V 600 E}$ melanoma. The V600 approval is based upon the inclusion of all $B R A F^{V 600}$ patients in the clinical trials (as determined by the cobas 4800 test), despite the fact that over $95 \%$ of patients in these trials were $B R A F^{V 600 E}$ patients and despite there being little evidence of efficacy outside this genotype.

\section{LGX818}

LGX818 is a type 1 BRAF inhibitor under investigation in phase I clinical trials, both as a single agent (NCT01436656) and in combination with other targeted therapies (NCT01543698).

\section{Dabrafenib}

\section{Drug design and preclinical activity}

Dabrafenib (GSK2118436) is a reversible and potent adenosine triphosphate-competitive inhibitor that selectively inhibits the $\mathrm{BRAF}^{\mathrm{V} 600 \mathrm{E}}$ kinase. The drug concentration required for $50 \%$ inhibition of $\mathrm{BRAF}^{\mathrm{V} 600 \mathrm{E}}$ kinase activity (IC50) is five times lower than that for $\mathrm{BRAF}^{\mathrm{wt}}$ or $\mathrm{CRAF}^{65}$ Preclinical data demonstrated that dabrafenib inhibits the MAPK pathway in $B R A F^{V 600 E}$ melanoma cells, leading to decreased proliferation and regression in xenograft mouse models. ${ }^{65}$

\section{Clinical trials}

Dabrafenib trial results are summarized in Table 2.

\section{Phase I trial (BREAK-I)}

Clinical trials of dabrafenib began in 2009. Initial results of the phase I trial were presented in $2010,{ }^{66,67}$ and the final results were reported in 2012. ${ }^{54}$ This trial included patients with solid tumors and Eastern Cooperative Oncology Group (ECOG) performance status of 0 or 1 . Initial inclusion criteria did not require the presence of $B R A F$ mutation, but this was 
Table 2 Summary of dabrafenib trial results

\begin{tabular}{|c|c|c|c|c|}
\hline Variable & BREAK- $\left.\right|^{54}$ & ${\text { BREAK- } 2^{69}}^{69}$ & BREAK-3 $^{3}$ & BREAK-MB $^{70}$ \\
\hline \multicolumn{5}{|l|}{ Patients (n) } \\
\hline Total & $156^{\mathrm{a}}$ & 92 & $187^{b}$ & 172 \\
\hline$B R A F^{\vee 600 E}$ & $|3|$ & 76 & 187 & 139 \\
\hline$B R A F^{\vee 600 K}$ & 18 & 16 & 0 & 33 \\
\hline Non-V600 BRAF mut & 4 & 0 & 0 & 0 \\
\hline \multicolumn{5}{|l|}{ Efficacy } \\
\hline \multirow[t]{3}{*}{ RR (\%) } & 56 (V600E) & 60 (V600E) & 53 & $31-36(\mathrm{~V} 600 \mathrm{E})^{c}$ \\
\hline & 22 (V600K) & 13 (V600K) & & $7-22(\mathrm{~V} 600 \mathrm{~K})^{\mathrm{c}}$ \\
\hline & 0 (other) & & & \\
\hline \multirow[t]{2}{*}{ PFS (months) } & 5.5 (V600E) & 6.2 (V600E) & 5.1 & 3.7 (V600E) \\
\hline & 5.5 (V600K) & 4.6 (V600K) & & I.8-3.7 (V600K) \\
\hline \multirow[t]{2}{*}{ OS (months) } & $\mathrm{N} / \mathrm{A}$ & $\mathrm{N} / \mathrm{A}$ & $\mathrm{N} / \mathrm{A}$ & $7.1-7.6$ (V600E) \\
\hline & & & & $3.7-5.1$ (V600K) \\
\hline \multicolumn{5}{|l|}{ Toxicity (\%) } \\
\hline $\mathrm{cuSCC} / \mathrm{KA}$ & 11 & 9 & 7 & 5 \\
\hline Pyrexia $^{d}$ & 4 & 3 & 3 & 6 \\
\hline
\end{tabular}

Notes: ${ }^{2}$ Number of melanoma patients treated; bnumber of dabrafenib patients treated; cinvestigator-assessed intracranial RR; 'dgrade 3 pyrexia or pyrexia defined as a serious adverse event.

Abbreviations: RR, response rate; PFS, progression-free survival; OS, overall survival; N/A, not available; cuSCC, cutaneous squamous cell carcinoma; KA, cutaneous keratoacanthoma.

mandated early during the escalation phases because of a lack of activity in three patients with $B R A F^{w t}$ tumors. The primary aim of the study was to establish safety, tolerability, and the recommended phase 2 dose (RP2D) of dabrafenib. Secondary objectives were to determine pharmacokinetic and pharmacodynamic profiles and tumor response. The trial began with dose titration from $12 \mathrm{mg}$ daily until the RP2D of $150 \mathrm{mg}$ twice a day (BID) was defined. After establishment of the dosage, three expanded cohorts were added: (1) those with metastatic melanoma, (2) those with asymptomatic untreated brain metastases ( $\geq 3 \mathrm{~mm}$ size), and (3) those with nonmelanoma solid tumors.

Of the 156 melanoma patients enrolled in the study, three were $B R A F^{w t}$, 131 were $B R A F^{V 600 E}$, 18 were $B R A F^{V 600 K}$, two were $B R A F^{K 601 E}$, one was $B R A F^{V 600 \_K 601 E}$, and one had an uncharacterized $B R A F$ mutation. The most common grade 2 or higher toxicities were cuSCC or keratoacanthoma (KA) (11\%), fatigue (8\%), and pyrexia (6\%). Palmoplantar hyperkeratoses and actinic keratoses were common (26\% and $10 \%$, respectively) but mild. Dose reductions occurred in $7 \%$ of patients, and $76 \%$ of patients had no drug-related adverse events of greater severity than grade 2. MTD was not reached at doses of up to $300 \mathrm{mg}$ BID; however, a minority of patients developed dose-limiting effects at $300 \mathrm{mg}$ BID (two of ten patients) and $200 \mathrm{mg}$ BID (three of 20 patients). A RP2D of $150 \mathrm{mg}$ BID was selected, since patients on $200 \mathrm{mg}$ BID showed minimal increase in drug exposure (area under the curve) with no increase in the proportion of patients with RECIST response, ${ }^{68}$ metabolic response assessed by positron emission tomography, or MAPK inhibition (measured by phospho-ERK expression on biopsy) and with the development of some dose-limiting events.

Of the $36 B R A F^{V 600}$ melanoma patients treated at RP2D, $25(69 \%)$ had a complete or partial response, and $18(50 \%)$ had a confirmed response. Of the $27 B R A F^{V 600 E}$ melanoma patients, 21 (78\%) had complete or partial responses, and 15 (56\%) had confirmed responses. Of the $18 B R A F^{V 600 K}$ patients receiving any dose of dabrafenib, seven (39\%) showed responses, and these responses were confirmed in four $(22 \%)$ patients. The median PFS was similar (approximately 5.5 months) in $B R A F^{V 600 E}$ and $B R A F^{V 600 K}$ patients. Nine $(90 \%)$ of the ten patients with $B R A F^{V 600}$ melanoma and previously untreated brain metastases showed a decrease in the size of their brain tumors, with four patients (40\%) achieving complete responses. No responses were seen in patients with $B R A F^{K 601 E}$ or $B R A F^{V 600 \_K 601 E}$ mutations.

Responses were also seen in $B R A F^{V 600 E}$ nonmelanoma tumors, including papillary thyroid cancer $(n=9)$ and nonsmall cell lung cancer $(n=1)$. Stable disease was seen in gastrointestinal stromal tumor $(\mathrm{n}=1)$ and ovarian cancer $(\mathrm{n}=1)$. Of the nine $B R A F^{V 600 E}$ colorectal cancer patients with known $B R A F$ status, one (11\%) had a partial response and seven $(78 \%)$ had stable disease.

In summary, the phase I study showed dabrafenib to be safe and tolerable, showed that dabrafenib demonstrated activity in $B R A F^{V 600 E}$ and $B R A F^{V 600 K}$ melanoma, and showed that dabrafenib was the first drug to show activity in melanoma metastases in the brain. 


\section{Phase II trial (BREAK-2)}

The single-arm, open-label, phase II trial included patients with $B R A F^{V 600 E}$ or $B R A F^{V 600 K}$ metastatic melanoma without prior therapy with BRAF or MEK inhibitors. ${ }^{69}$ Patients with current or prior brain metastases were excluded. The primary objective was to determine the response rate in $B R A F^{V 600 E}$ patients, with secondary objectives including response rate in $B R A F^{V 600 K}$ patients, duration of response, PFS, OS, safety, and tolerability.

In 92 enrolled patients (76 BRAF V600E, $\left.16 B R A F^{V 600 K}\right)$, dabrafenib was dosed at $150 \mathrm{mg}$ BID. Most patients (63\%) had American Joint Committee on Cancer stage M1c disease, and $84 \%$ had received prior therapy for metastatic disease. Adverse events were common but rarely led to dose reduction (14\%) or discontinuation (1\%). Most frequent (ranging from $15 \%$ to $33 \%$ ) toxicities of any grade were arthralgia, hyperkeratosis, pyrexia, fatigue, headache, nausea, and skin papillomas. Serious adverse events occurred in $27 \%$ of patients and included cuSCCs (9\%), basal-cell carcinomas (4\%), and pyrexia (3\%).

Response rates were impressive in $B R A F^{V 600 E}$ patients (7\% complete response, $53 \%$ partial response) but less so in $B R A F^{V 600 K}$ patients ( $0 \%$ complete response, $13 \%$ partial response). A minority of patients (16\% BRAF V600E, $31 \%$ $B R A F^{V 600 K}$ ) appeared to derive no benefit from treatment and had progressive disease at first assessment. The median PFS was longer (27 weeks in $B R A F^{V 600 E}$ patients, 20 weeks in $B R A F^{V 600 K}$ patients) than that reported for standard chemotherapies.

\section{Phase III trial (BREAK-3)}

The phase III trial recruited patients with stage IV or unresectable stage IIIC $B R A F^{V 600 E}$ melanoma, with no prior therapy for advanced disease (apart from interleukin-2 in one patient), and an ECOG performance status of 0 or $1 .{ }^{3}$ Patients with a history of brain metastases required demonstration of stability for 3 months after local treatment (surgery, stereotactic radiotherapy). The trial was conducted in a 3:1 randomized, open-label design, with patients receiving either dabrafenib $150 \mathrm{mg}$ BID or DTIC (1000 mg/m² every 3 weeks), with crossover to dabrafenib upon disease progression in those receiving DTIC. The primary end point was investigatorassessed PFS, with secondary end points including PFS, as assessed by an independent review committee (IRC); OS; objective response rate by revised RECIST guidelines (version 1.1), ${ }^{63}$ as determined by an investigator and an IRC; PFS after crossover from DTIC to dabrafenib; duration of response; quality of life; safety; and tolerability.
Initial results were reported in mid-2012. ${ }^{3}$ Two hundred and fifty $B R A F^{V 600 E}$ patients were enrolled, 187 in the dabrafenib arm and 63 in the DTIC arm. The two treatment groups were well balanced. Most patients receiving dabrafenib had an ECOG performance status of $0(66 \%)$, American Joint Committee on Cancer stage M1c disease (66\%), and a normal serum lactate dehydrogenase (LDH) level (64\%). Response, outcome, and toxicity data were presented as of data cutoff on December 19, 2011, at which time $57 \%$ of patients in the dabrafenib group were still receiving treatment, with a median follow-up of 4.9 months.

Investigator-assessed estimated median PFS in the dabrafenib group was 5.1 months, compared with 2.1 months in the DTIC group, and the hazard ratio (HR) for progression was 0.30 (95\% confidence interval [CI], 0.18-0.51; $P<0.0001)$. PFS as assessed by an IRC was 6.7 months for dabrafenib and 2.9 months for DTIC (HR, 0.35; 95\% CI, 0.20-0.61). The improved PFS with dabrafenib was seen in all subgroups (ECOG performance statuses 0 and 1; normal and elevated LDH levels; age $<65$ years and $\geq 65$ years; male and female; disease stages IIIC, M1a, M1b, and M1c). OS data were not mature, and mortality rates were only $11 \%$ and $14 \%$ in the dabrafenib and DTIC arms, respectively. Twenty-two percent of DTIC patients were still receiving treatment, $44 \%$ having crossed over to dabrafenib after disease progression. Confirmed responses by an IRC were seen in $50 \%$ of dabrafenib patients ( $3 \%$ complete, $47 \%$ partial), and $6 \%$ of DTIC patients ( $2 \%$ complete, $4 \%$ partial). The median time to response in the dabrafenib group was 6.3 weeks (the time of first tumor assessment). The estimated median duration of response was 5.5 months. Twenty-eight patients in the DTIC group progressed and then crossed over to dabrafenib by the time of trial analysis; of these patients, $13(46 \%)$ achieved subsequent partial responses.

Toxicities were similar to those seen in the early-phase trials, the most common being cutaneous manifestations (hyperkeratosis, papillomas, palmar-plantar erythrodysesthesia), pyrexia, fatigue, headache, and arthralgia. Twelve (6\%) of 187 patients had cuSCCs/KAs, three patients (2\%) developed new primary melanomas, and phototoxicity was rare $(3 \%)$. Dose reductions occurred in $28 \%$ of patients, and $3 \%$ of patients permanently discontinued therapy because of toxicity.

OS data are expected to be similar to the data seen with vemurafenib, ${ }^{61,62}$ in that the improved PFS will parallel improved OS. The difference in OS between the treatment arms will no doubt be diminished because of crossover in the DTIC group, but nevertheless it should remain, as in the vemurafenib trial. 


\section{Phase II brain metastases trial (BREAK-MB)}

Following the impressive results seen in the ten patients enrolled in the phase I trial with active brain metastases, a dedicated phase II study was conducted to further examine the effect of dabrafenib in those with untreated, or previously treated but relapsed, brain metastases..$^{70}$ This trial enrolled patients with $B R A F^{V 600 E / K}$ melanoma, no prior MAPK therapy (BRAF or MEK inhibitors), asymptomatic brain metastases, ECOG performance status of 0-1, and up to two prior lines of systemic therapy for metastatic disease. Patients were allowed corticosteroids if they were receiving a stable or decreasing dose over 3 weeks prior to enrollment, and at least one brain metastasis needed to be between 0.5 and $4 \mathrm{~cm}$ in size to be a measurable target lesion. The presence of leptomeningeal disease excluded patients from the study. The primary end point was the investigator-assessed overall intracranial response rate (OIRR) in $B R A F^{V 600 E}$ patients, and secondary end points were, in $B R A F^{V 600 K}$ patients, the OIRR and, in all patients, the overall response rate, degree of intracranial and overall response, PFS, and OS.

One hundred and seventy-two patients were enrolled in two cohorts: (1) those without prior local treatment for brain metastases $(n=89)$ and (2) those with brain metastases previously treated with local therapy (surgery, stereotactic radiosurgery, and/or whole brain radiotherapy) but with subsequent intracranial progression $(n=83)$. The two cohorts were unbalanced with regard to ECOG performance status, the number of target brain metastases, and prior therapies received, and were therefore not directly comparable. However, most patients in both cohorts had an ECOG performance status of 0 , an elevated serum LDH level, fewer than four target brain metastases, and had received previous chemo- or immunotherapy.

Dabrafenib was well tolerated, and the toxicity was similar to that seen in the aforementioned studies, with the exception of intracranial hemorrhage, which occurred in $6 \%$ of patients. Hemorrhage occurred in both cohorts, in both responding and progressive lesions; it did not appear higher than the background rate, ${ }^{71,72}$ and it was thought to be related to the patient population and their intracranial disease rather than to the drug.

Responses were seen in both cohorts, and in both $B R A F^{V 600 E}$ and $B R A F^{V 600 K}$ genotypes. In $B R A F^{V 600 E}$ melanoma patients, the investigator-assessed OIRRs in untreated patients and in previously treated patients were $39 \%$ and $31 \%$, respectively, while the overall response rates were $38 \%$ and $31 \%$, respectively. The intracranial disease control rate (defined as complete + partial response + stable disease) was $80 \%-90 \%$.
In $B R A F^{V 600 E}$ patients in both cohorts, the median PFS was 16 weeks and the median OS was 31-33 weeks. In untreated and previously treated $B R A F^{V 600 K}$ melanoma patients $(\mathrm{n}=33)$, OIRRs were $7 \%$ and $22 \%$, respectively, and disease control rates were $33 \%$ and $50 \%$, respectively. The median PFS ranged from 8 to 16 weeks, and the median OS ranged from 16 to 22 weeks, the difference between the cohorts being most likely due to the small numbers involved. No data were available regarding the causes of disease progression, but the causes may be inferred from the results of a single institution study of 20 patients that reported that progression occurred in the brain alone in $30 \%$ of patients, in the brain and extracranially in $40 \%$, and extracranially alone (with ongoing intracranial control) in $30 \%{ }^{73}$

The results of this trial were impressive when compared with studies of systemic agents, which report response rates of less than $10 \%$, PFS of less than 2 months, and OS of 3-5 months. ${ }^{11,74-76}$ The findings suggested that dabrafenib may be an effective adjunct for treatment of brain metastases (alongside surgery and radiotherapy), and that it warrants consideration as first-line therapy in patients with brain metastases, particularly if the brain metastases are multiple or concurrent with advanced extracranial disease.

\section{Differences between dabrafenib and vemurafenib}

Dabrafenib and vemurafenib are both selective type $1 \mathrm{BRAF}$ inhibitors with proven efficacy in BRAFV600E metastatic melanoma. They share many attributes, including clinical activity and class-defined toxicity; however, differences between the drugs exist in regard to RAF kinase inhibition, toxicities, and prospective evidence for activity in nonV600E BRAF melanoma and in brain metastases.

\section{Selective inhibition of RAF kinases and MTD}

Vemurafenib and dabrafenib are more selective for BRAF ${ }^{\text {mut }}$ than other kinases, including BRAFwt. Vemurafenib has similar potency for inhibition of CRAF (IC50 $48 \mathrm{nM}$ ) and BRAF ${ }^{\mathrm{V} 600 \mathrm{E}}$ (IC50 $31 \mathrm{nM}$ ), while dabrafenib has less potency for inhibition of CRAF (IC50 $5 \mathrm{nM}$ versus $0.6 \mathrm{nM}$ for BRAF ${ }^{\mathrm{V} 600 \mathrm{E}}$ ). ${ }^{53,66}$ The ratios of IC50 for CRAF and BRAF ${ }^{\mathrm{V} 600 \mathrm{E}}$ compared with that for $\mathrm{BRAF}^{\mathrm{wt}}$ are 0.5 and 0.3 , respectively, for vemurafenib ${ }^{53}$ and 0.4 and 0.05 , respectively, for dabrafenib, ${ }^{66}$ indicating that dabrafenib is a more selective inhibitor of $\mathrm{BRAF}^{\mathrm{V} 600 \mathrm{E}}$ than vemurafenib. Furthermore, dabrafenib has similar potency for inhibition of BRAF ${ }^{\mathrm{V} 600 \mathrm{E}}$ and $\mathrm{BRAF}^{\mathrm{V} 600 \mathrm{~K}} .^{54}$ Dabrafenib did not reach MTD in the phase I trial, ${ }^{54}$ and its therapeutic dose 
was based on a minimum increase in drug exposure, clinical response, and MAPK inhibition at the next higher dose level (200 mg BID). Vemurafenib reached MTD (960 mg BID) in its phase I trial, ${ }^{4}$ with unacceptable toxicities developing in four of six patients (grade 3 rash and arthralgia) at the next higher dose level (1120 mg BID).

\section{Efficacy in non-V600E BRAF melanoma patients}

Vemurafenib has only been studied prospectively in $B R A F^{V 600 E}$ patients, since the companion diagnostic test used to define study entry (cobas 4800) is highly sensitive and specific for the $B R A F^{V 600 E}$ mutation. Definitive conclusions regarding response rates, PFS, and OS in vemurafenib-treated $B R A F^{V 600 K}$ melanoma cannot be derived because of the small number of patients in the phase II and III trials that were retrospectively characterized as $B R A F^{V 600 K}$. A clinical trial to evaluate vemurafenib activity in patients with non-V600E exon $15 B R A F$ mutations is under way (NCT01586195). Dabrafenib was prospectively tested in non-V600E $B R A F$ genotypes in the phase I and II trials, and there is good evidence for its activity in $B R A F^{V 600 K}$ melanoma.

\section{Efficacy in BRAF ${ }^{V 600 E}$ melanoma patients}

It is difficult to compare the relative clinical efficacy of dabrafenib and vemurafenib in $B R A F^{V 600 E}$ melanoma patients, as no head-to-head trials have been conducted. Furthermore, mature outcome data regarding PFS and OS are only available at this time for vemurafenib. From the available data, it appears that the trials of each drug included patients with similar disease characteristics. Based on this, rough comparisons can be made, and these suggest the drugs share similar response rates (approximately 50\%) in $B R A F^{V 600 E}$ patients. The phase III trials of dabrafenib and vemurafenib trials both reported similar PFS results at early time points (5.1 and 5.3 months, respectively). The median PFS with vemurafenib is 6.9 months, and mature PFS data from dabrafenib-treated patients are yet to be reported.

\section{Intracranial activity}

Dabrafenib has proven intracranial activity, and phase I and II trial data support its clinical efficacy in $B R A F^{V 600 E}$ and $B R A F^{V 600 K}$ patients with brain metastases. Vemurafenib is expected to exhibit similar intracranial activity; however, at present, results are only available from a small phase I study of $B R A F^{V 600 E}$ patients. ${ }^{64}$ A larger phase II study in cobas-positive $\left(B R A F^{V 600 E}\right)$ melanoma patients is ongoing (NCT01378975).

\section{Toxicity}

Trials of dabrafenib and vemurafenib show differences in the incidences of toxicities (Tables 3 and 4). Cutaneous toxicities such as rash, hyperkeratosis, cuSCC, and KA occur with both drugs but have been reported to occur to a lesser degree with dabrafenib. Of note, cuSCCs occur in 19\% of patients with vemurafenib ${ }^{61}$ and in only $5 \%$ of patients with dabrafenib. ${ }^{3}$ Other toxicities such as arthralgia and fatigue also appear to occur at a higher rate and grade with vemurafenib. Drug-specific toxicities include photosensitivity and hepatitis with vemurafenib ${ }^{61}$ and pyrexia with dabrafenib. ${ }^{3}$ The need for dose reduction or interruption due to toxicity was approximately $30 \%-40 \%$ for both drugs, with only a minimal number of patients treated with either drug, permanently discontinuing therapy because of toxicity.

The higher incidence of class-like toxicities seen with vemurafenib than with dabrafenib is likely to be due to several factors: differences in drug dose (vemurafenib is dosed at one level below MTD, while the MTD for dabrafenib is unknown); RAF inhibitor potency (BRAF ${ }^{\text {mut }}$ and CRAF); histopathologic assessment of cutaneous lesions (central review in the phase I and II studies of vemurafenib only); classification and reporting of toxicity; and different geographic populations enrolled in the studies (eg, differences in UV exposure affecting inherent cuSCC risk). Analysis of the mechanisms of cutaneous toxicities (notably, cuSCC) suggest that in $B R A F^{w t}$ cells, BRAF inhibitors cause formation of RAF dimers that lead to signaling through CRAF, particularly in those with an upstream mutation such as $R A S .{ }^{77-79}$ Studies have shown frequent mutations in $H R A S$, and to a lesser extent $N R A S$, in benign and malignant (cuSCCs) proliferative lesions resected from patients on BRAF inhibitors,

Table 3 Comparative toxicities of vemurafenib and dabrafenib

\begin{tabular}{lll}
\hline Toxicity (all grades) & Vemurafenib & Dabrafenib \\
\hline Hyperkeratosis & Present & $51 \%$ \\
Palmoplantar hyperkeratosis/ & $10 \%^{\mathrm{a}}$ & $21 \%$ \\
erythrodysesthesia & & \\
cuSCC/KA & $30 \%$ & $7 \%$ \\
Rash & $41 \%$ & $22 \% \mathrm{~b}$ \\
Arthralgia & $56 \%$ & $16 \%$ \\
Pyrexia & $\mathrm{NR}$ & $15 \%$ \\
Photosensitivity & $41 \%$ & $3 \%$ \\
Hepatitis & $26 \%$ & $\mathrm{NR}$ \\
Dose reduction or interruption & $38 \%$ & $28 \% \mathrm{c}$ \\
Discontinuation & $7 \%$ & $3 \%$ \\
\hline
\end{tabular}

Notes: ${ }^{2}$ Data from the phase II vemurafenib trial; 60 bdata from the phase II dabrafenib trial; ${ }^{69}$ conly dose reduction data presented. Toxicity data are from phase III clinical trials. ${ }^{3,61,62,122}$ Hyperkeratosis and rash have been poorly defined or classified in trials, making comparisons difficult.

Abbreviations: cuSCC, cutaneous squamous cell carcinoma; KA, cutaneous keratoacanthoma; NR, not reported. 
Table 4 Outcome measures and grade $3 / 4$ toxicities with BRAF and MEK inhibitors

\begin{tabular}{|c|c|c|c|c|}
\hline & Vemurafenib $^{61,62}$ & Dabrafenib $^{3}$ & Trametinib $^{105}$ & CombiDT $(150 / 2)^{106}$ \\
\hline \multicolumn{5}{|l|}{ Outcomes } \\
\hline $\mathrm{RR}(\%)$ & 57 & 53 & 22 & 76 \\
\hline DCR (\%) & 97 & 95 & 78 & 100 \\
\hline PFS (months) & 6.9 & 5.1 & 4.8 & 9.4 \\
\hline OS (months) & 13.6 & NA & NA & NA \\
\hline \multicolumn{5}{|l|}{ Toxicity (\%) } \\
\hline cuSCC & 19 & 5 & 0 & $5^{a}$ \\
\hline $\mathrm{KA}$ & 10 & 2 & 0 & \\
\hline Hyperkeratosis & I & 3 & 0 & 0 \\
\hline Rash & 9 & 0 & 9 & 0 \\
\hline Pyrexia & 0 & 3 & 0 & 5 \\
\hline \multirow[t]{3}{*}{ Other } & I0, hepatitis & & 12 , hypertension & \\
\hline & & & 7, cardiac & \\
\hline & & & I, ocular & \\
\hline
\end{tabular}

Note: ${ }^{a}$ cuSCC and KA combined; only vemurafenib has mature outcome data at this stage.

Abbreviations: CombiDT, combined dabrafenib and trametinib; I50/2, dose of dabrafenib I50 mg BID and trametinib 2 mg daily; RR, response rate (complete + partial response); DCR, disease control rate (complete + partial response + stable disease); PFS, progression-free survival; OS, overall survival; NA, not assessable; cuSCC, cutaneous squamous cell carcinoma; KA, cutaneous keratoacanthoma.

at higher rates than in lesions from patients not treated with inhibitors. ${ }^{80-83}$

Pyrexia is a specific toxicity seen with dabrafenib that may respond to steroids. The mechanisms underlying dabrafenib-associated pyrexia are poorly understood and require further investigation.

Photosensitivity occurs with vemurafenib, it may develop despite minimal UV exposure, and it is often a cause of significant morbidity. Across the phase I-III trials with vemurafenib, photosensitivity occurred in $40 \%-50 \%$ of patients (all grades), and 3\% were grade 3.,60,61 The only dabrafenib trial reporting photosensitive reactions was the phase III study, in which $3 \%$ of patients had low-grade toxicity, with no grade 3 events. ${ }^{3}$ Photosensitivity was not reported in the phase I and II trials of dabrafenib and is therefore considered to be a vemurafenib-specific toxicity.

Hepatitis is another toxicity that appears to be specific to vemurafenib. It has not been reported in any trials with dabrafenib. Seventeen percent of patients in the phase II study of vemurafenib had elevated liver enzymes, and in $10 \%$ of patients this was grade 3 or higher, leading to dose reduction or permanent discontinuation in all cases. ${ }^{60}$

\section{Limitations of dabrafenib and future directions for treatment of patients with metastatic melanoma}

Dabrafenib has proven activity in patients with $B R A F^{V 600 E}$ and $B R A F^{V 600 K}$ metastatic melanoma, including those with brain metastases. The most common toxicities are cutaneous and mild, rarely leading to discontinuation. Compared with previous systemic therapies for metastatic melanoma, dabrafenib is highly effective, with high response rates and an improved survival; however, acquired resistance remains the greatest limitation. Combinations of dabrafenib with other agents, including immunotherapies and other targeted agents, hold great promise for improved treatment of melanoma patients.

\section{Variable response and resistance to BRAF inhibitors} There is a broad spectrum of response to dabrafenib and vemurafenib therapy. A minority of patients $(3 \%-5 \%)$ demonstrate no overall RECIST response (progressive disease at first assessment), while a few (3\%-5\%) show complete responses with long-term remission and are possibly cured..$^{3,61,62}$ However, the vast majority of patients have rapid, incomplete, and heterogeneous early treatment responses (shrinkage of tumors and decline in tumor metabolism assessed by positron emission tomography), ${ }^{84,85}$ with subsequent overall disease progression at variable time points during treatment. This phenomenon occurs as a result of both primary and acquired resistance, and inter- and intratumoral heterogeneity ${ }^{86,87}$ - features that present major challenges for drug development.

To date, there are no biomarkers that accurately predict clinical outcomes (response, PFS, and OS). Studies assessing baseline pretreatment tissue and tissue taken early during treatment (approximately 2 weeks after starting therapy) that have examined many markers of MAPK pathway activity and other signaling pathways have shown little correlation with clinical outcome. ${ }^{88-91}$ The best current indicator of outcome is the $B R A F$ genotype, with $B R A F^{V 600 E}$ melanoma patients consistently performing better than patients with non-V600E $B R A F^{V 600}$ melanomas. $^{54,69}$ 
A small number of studies have been performed in pretreatment tissues and cell lines to assess for mechanisms of primary resistance. These suggest that amplification of the CCND1 gene (encoding cyclin D1), ${ }^{92}$ and stromal expression of hepatocyte growth factor, ${ }^{93}$ may play a role in conferring primary resistance to BRAF inhibitor treatment. Most attention, however, has been focused on understanding the specific mechanisms behind the development of acquired resistance to BRAF inhibitors. Biopsies of progressing tumors from patients, when compared with corresponding pretreatment samples, usually demonstrate reactivation of the MAPK pathway. This has been shown to occur because of acquired aberrations including amplification ${ }^{94}$ and splice variation ${ }^{95}$ of $B R A F$, RAF isoform switching, ${ }^{96,97}$ new mutations in $N R A S^{98}$ and $M E K,{ }^{99}$ and increased expression of COT, ${ }^{100}$ a partner kinase (Figure 1). A minority of cases do not demonstrate MAPK reactivation but do show increased signaling through other pathways (such as the PI3K pathway); these appear to be a result of increased expression of growth factor receptors such as insulin-like growth factor 1 receptor $^{96}$ and plateletderived growth factor receptor beta. ${ }^{98}$ To date, it appears that no single mechanism predominates. However, changes to the drug-binding site in the BRAF protein (a common mechanism of acquired resistance with other targeted therapies ${ }^{101,102}$ ) have not been reported.

\section{Other targeted agents}

MEK inhibitors have also been studied in $B R A F^{m u t}$ melanoma. They were designed to inhibit MAPK signaling at a level downstream of BRAF. The development of MEK inhibitors began prior to that of BRAF inhibitors, and MEK inhibitors showed limited effect in initial trials in melanoma patients without unknown BRAF status. Impressive results have been obtained in more recent trials that were conducted in $B R A F^{\text {mut }}$ melanoma patients.

Trametinib (GSK1120212) is the best-studied MEK inhibitor in $B R A F^{m u t}$ melanoma. A phase I trial demonstrated significant activity in $B R A F^{m u t}$ melanoma, with little activity in $B R A F^{w t}$ patients, ${ }^{103}$ while a phase II study in $B R A F^{V 600 E / K}$ patients with or without prior BRAF inhibitor treatment demonstrated no response when given after BRAF inhibitor failure. ${ }^{104}$ Initial reports from the recent first-line phase III trial show a response rate of $22 \%$ and a median PFS of 4.8 months (Table 4), compared with an $8 \%$ response and 1.4 months' PFS in the chemotherapy (DTIC or paclitaxel) arm. OS data were immature, but the HR for progression or death was $0.54(95 \% \mathrm{CI}, 0.32-0.92 ; P=0.01)$. The 6-month OS rate in the intention-to-treat population was $81 \%$ in the trametinib group and 67\% in the chemotherapy group, despite $47 \%$ of patients in the chemotherapy group crossing over to trametinib upon disease progression. ${ }^{105}$ Toxicities included MEK inhibitor class-like effects such as rash (including acneiform rash), hypertension, diarrhea, edema, transient mild cardiac dysfunction, rare ocular toxicity (chorioretinopathy), and creatine kinase elevation. Most toxicities were mild and did not require drug discontinuation.

The results of the trial suggest that trametinib was not as effective as dabrafenib (or vemurafenib) therapy but still showed impressive single-agent activity. Monotherapy with trametinib rather than dabrafenib would only appear prudent for those who could not tolerate dabrafenib because of toxicity.

\section{Combination dabrafenib and trametinib}

An effective strategy to build upon the successes seen with dabrafenib and trametinib monotherapies has been to combine these agents (CombiDT), with the goal of further improving response rates and delaying resistance. As both drugs target the MAPK pathway, combined blockade may circumvent or delay acquired resistance because of reactivation of the MAPK pathway. Combining the two drugs may also reduce the toxicities of each drug when given individually (especially cutaneous toxicity from dabrafenib).

The initial phase I/II trial involved $B R A F^{V 600 E / K}$ metastatic melanoma patients across multiple cohorts. ${ }^{106}$ These cohorts included a drug-drug interaction cohort (Part A), dose escalation and expansion cohorts (Part B), and a large randomized cohort (Part $\mathrm{C}$ ). In Part $\mathrm{C}$, patients were randomized 1:1:1 to CombiDT at a dose of dabrafenib $150 \mathrm{mg}$ BID and trametinib $1 \mathrm{mg}$ daily, CombiDT at a dose of dabrafenib $150 \mathrm{mg}$ BID and trametinib $2 \mathrm{mg}$ daily (150/2), or dabrafenib monotherapy (150 mg BID). Analysis of data from the randomized phase II (Part C) trial in BRAF/MEK inhibitor-naïve melanoma patients reported a response rate of $76 \%$ in those receiving CombiDT at the $150 / 2$ dose, which was significantly higher than that in patients receiving dabrafenib monotherapy $(54 \% ; P=0.026)$. More important, the median PFS of 9.4 months in those receiving CombiDT at the 150/2 dose was significantly longer than that in the dabrafenib monotherapy arm (5.8 months; HR, 0.39; $P<0.0001) .{ }^{106}$ These results are better than those reported in the previous phase III dabrafenib and trametinib monotherapy trials (Table 4). ${ }^{3,61,62,105}$ Additionally, in the Part B expansion cohort of patients with prior disease progression during BRAF inhibitor treatment, an impressive $19 \%$ response rate was seen with CombiDT therapy. ${ }^{107}$ 
Toxicities with this combination were generally mild. Notably, cutaneous toxicities such as hyperkeratosis, cuSCC, $\mathrm{KA}$, and rash were greatly reduced, and there appeared to be a reduction in diarrhea and hypertension (trametinib-related toxicities) compared with single-agent trametinib trials. ${ }^{105}$ The most common toxicity was fever, occurring in approximately $70 \%$ of patients ( $5 \%$ grade $3 / 4$ ) of those patients receiving CombiDT at the 150/2 dose-much more frequent than with dabrafenib monotherapy. ${ }^{3,106}$ The mechanisms underlying the pyrexia remain incompletely understood, but pyrexia generally occurs early, is often an isolated episode, can be managed with brief dose interruption and corticosteroid prophylaxis (in recurrent cases), and does not require dose reduction. ${ }^{108}$ Furthermore, pyrexia does not appear to be related to disease burden or treatment response. ${ }^{108}$

Two phase III trials of CombiDT are under way, comparing CombiDT at the 150/2 dose with dabrafenib (NCT01584648) and vemurafenib (NCT01597908) monotherapies. The results of these studies may lead to replacement of BRAF inhibitor monotherapy by combination BRAF and MEK inhibitor therapy as the first-line treatment for $B R A F^{m u}$ melanoma.

\section{Other combinations}

Further combination treatments are being proposed as an increased understanding of the factors involved in tumor response and resistance develops, as well as the development of new drugs that target pathways in the melanoma cell outside the MAPK axis.

Immunotherapies, which directly manipulate T-cell activation in the hope of improving antitumor immunity, have recently emerged as an important form of treatment for metastatic melanoma. The first agent with proven activity, ipilimumab, is an anti-CTLA-4 antibody that binds to and inhibits the CTLA-4 T-cell receptor, resulting in sustained but nonspecific T-cell activation. Two phase III clinical trials have now been completed in the first- ${ }^{109}$ and second-line settings, ${ }^{110}$ with reported response rates of $11 \%-15 \%$, a median PFS of 2.8 months, and a median OS of 10-11 months. One- and two-year survival was approximately $47 \%$ and $26 \%$, respectively - a $10 \%$ increase over the control arms. Activity has also been demonstrated in patients with small and asymptomatic brain metastases. ${ }^{111}$ Early-phase trials of other immune agents, including anti-PD- ${ }^{112,113}$ and anti-PD-L1 antibodies, ${ }^{114}$ have shown these immune agents appear to be more active and less toxic than ipilimumab.

Combining BRAF inhibitors with immunotherapy would appear to be safe and effective, given differences in their modes of action and toxicity profiles. Translational evidence in support of this approach is also robust, with evidence that BRAF inhibition leads to increased expression of melanoma differentiation antigens and an influx of tumor-infiltrating lymphocytes ${ }^{88,115} \mathrm{~A}$ trial of vemurafenib and ipilimumab has commenced (NCT01400451), and it is expected that dabrafenib will be combined with immunotherapy in the future.

Combinations of drugs targeting other cell signaling pathways (eg, BRAF inhibitors plus PI3K inhibitors), based on discoveries of BRAF inhibitor resistance mechanisms, have entered clinical trials.

\section{Adjuvant therapy}

Given the impressive activity of dabrafenib and vemurafenib in $B R A F^{m u t}$ metastatic melanoma, it is expected that they may have an important role to play in adjuvant therapy of earlystage melanoma. At present, there is little therapy available for patients with early-stage melanoma. The only approved treatment, interferon, is highly toxic, requires a prolonged duration of treatment, and has little impact on OS. ${ }^{116,117} \mathrm{~A}$ trial of adjuvant ipilimumab versus placebo has completed recruitment and results are expected in 2013 (NCT00636168). The adjuvant trial of vemurafenib versus placebo in resected stage IIC/III melanoma (NCT01667419) has commenced, and a trial of CombiDT versus placebo in resected stage III melanoma (NCT01682083) will open soon.

\section{Conclusion}

Fifty percent of cutaneous melanomas have mutations in $B R A F$. These mutations result in highly elevated activity of the MAPK signaling pathway, which mediates cell growth and survival. Dabrafenib effectively inhibits $B R A F^{\text {mut }}$ kinase, thereby inhibiting MAPK signaling in $B R A F^{m u t}$ melanoma. In patients with $B R A F^{m u t}$ metastatic melanoma, dabrafenib confers high response rates and an improved PFS over chemotherapy, with a mild, manageable toxicity profile; however, the duration of benefit is usually brief because of the development of acquired resistance. It is expected that new combination therapies targeting multiple components of cell signaling, such as CombiDT, will provide higher response rates and more durable clinical benefit than dabrafenib monotherapy.

\section{Summary points}

- BRAF mutations occur in $50 \%$ of melanomas; $70 \%-90 \%$ are $\mathrm{V} 600 \mathrm{E}$ and $10 \%-30 \%$ are $\mathrm{V} 600 \mathrm{~K}$.

- Selective (type 1) BRAF inhibitors (dabrafenib, vemurafenib) target mutant BRAF kinase and inhibit the MAPK pathway. 
- Both drugs provide high response rates and rapid modes of action, but both are limited by the rapid development of acquired resistance.

- Both dabrafenib and vemurafenib are effective in treating $B R A F^{V 600 E}$ melanoma patients, with prospective evidence of dabrafenib activity in $B R A F^{V 600 K}$ melanoma and in those with brain metastases.

- Both drugs have similar toxicity profiles; dabrafenib appears to have less cutaneous and hepatic toxicity than vemurafenib, but is associated with pyrexia.

- Emerging combination strategies, such as the CombiDT, are designed to improve response and delay resistance.

\section{Disclosure}

The authors acknowledge the following support and funding: A Menzies received travel support from Roche and GlaxoSmithKline; G Long received institutional research support from Roche and travel support from Roche and Bristol-Myers Squibb. G Long is a consultant advisor to Roche, GlaxoSmithKline, Bristol-Myers Squibb, and Amgen; R Murali reports no conflicts of interest in this work.

\section{References}

1. Balch CM, Gershenwald JE, Soong SJ, et al. Final version of 2009AJCC melanoma staging and classification. J Clin Oncol. 2009;27(36): 6199-6206.

2. Davies H, Bignell GR, Cox C, et al. Mutations of the BRAF gene in human cancer. Nature. 2002;417(6892):949-954.

3. Hauschild A, Grob JJ, Demidov LV, et al. Dabrafenib in BRAF-mutated metastatic melanoma: a multicentre, open-label, phase 3 randomised controlled trial. Lancet. 2012;380(9839):358-365.

4. Flaherty KT, Puzanov I, Kim KB, et al. Inhibition of mutated, activated BRAF in metastatic melanoma. N Engl J Med. 2010;363(9):809-819.

5. Neagu M, Constantin C, Tanase C. Immune-related biomarkers for diagnosis/prognosis and therapy monitoring of cutaneous melanoma. Expert Rev Mol Diagn. 2010;10(7):897-919.

6. Hauschild A, Agarwala SS, Trefzer U, et al. Results of a phase III, randomized, placebo-controlled study of sorafenib in combination with carboplatin and paclitaxel as second-line treatment in patients with unresectable stage III or stage IV melanoma. J Clin Oncol. 2009;27(17): 2823-2830.

7. Flaherty KT, Lee SJ, Schuchter LM, et al. Final results of E2603: a double-blind, randomized phase III trial comparing carboplatin (C)/ paclitaxel (P) with or without sorafenib (S) in metastatic melanoma [abstract]. J Clin Oncol. 2010;28(15 Suppl):Abstract 8511.

8. Middleton MR, Grob JJ, Aaronson N, et al. Randomized phase III study of temozolomide versus dacarbazine in the treatment of patients with advanced metastatic malignant melanoma. J Clin Oncol. 2000;18(1): $158-166$.

9. Eggermont AM, Schadendorf D. Melanoma and immunotherapy. Hematol Oncol Clin North Am. 2009;23(3):547-564, ix-x.

10. Patel PM, Suciu S, Mortier L, et al; EORTC Melanoma Group. Extended schedule, escalated dose temozolomide versus dacarbazine in stage IV melanoma: final results of a randomised phase III study (EORTC 18032). Eur J Cancer. 2011;47(10):1476-1483.

11. Avril MF, Aamdal S, Grob JJ, et al. Fotemustine compared with dacarbazine in patients with disseminated malignant melanoma: a phase III study. J Clin Oncol. 2004;22(6):1118-1125.
12. Atkins MB, Lotze MT, Dutcher JP, et al. High-dose recombinant interleukin 2 therapy for patients with metastatic melanoma: analysis of 270 patients treated between 1985 and 1993. J Clin Oncol. 1999;17(7): 2105-2116.

13. Brose MS, Volpe P, Feldman M, et al. BRAF and RAS mutations in human lung cancer and melanoma. Cancer Res. 2002;62(23):6997-7000.

14. Wellbrock C, Ogilvie L, Hedley D, et al. V599EB-RAF is an oncogene in melanocytes. Cancer Res. 2004;64(7):2338-2342.

15. Wu J, Rosenbaum E, Begum S, Westra WH. Distribution of BRAF T1799A(V600E) mutations across various types of benign nevi: implications for melanocytic tumorigenesis. Am J Dermatopathol. 2007;29(6):534-537.

16. Curtin JA, Fridlyand J, Kageshita T, et al. Distinct sets of genetic alterations in melanoma. N Engl J Med. 2005;353(20):2135-2147.

17. Hacker E, Hayward NK, Dumenil T, James MR, Whiteman DC. The association between MC1R genotype and BRAF mutation status in cutaneous melanoma: findings from an Australian population. $J$ Invest Dermatol. 2009;130(1):241-248.

18. Houben R, Becker JC, Kappel A, et al. Constitutive activation of the RAS-RAF signaling pathway in metastatic melanoma is associated with poor prognosis. J Carcinog. 2004;3(1):6.

19. Liu W, Kelly JW, Trivett M, et al. Distinct clinical and pathological features are associated with the BRAF(T1799A(V600E)) mutation in primary melanoma. J Invest Dermatol. 2006;127(4):900-905.

20. Viros A, Fridlyand J, Bauer J, et al. Improving melanoma classification by integrating genetic and morphologic features. PLoS Med. 2008; $5(6): \mathrm{e} 120$

21. Thomas NE, Edmiston SN, Alexander A, et al. Number of nevi and earlylife ambient UV exposure are associated with BRAF-mutant melanoma. Cancer Epidemiol Biomarkers Prev. 2007;16(5):991-997.

22. Long GV, Menzies AM, Nagrial AM, et al. Prognostic and clinicopathologic associations of oncogenic BRAF in metastatic melanoma. J Clin Oncol. 2011;29(10):1239-1246.

23. Jakob JA, Bassett RL Jr, Ng CS, et al. NRAS mutation status is an independent prognostic factor in metastatic melanoma. Cancer. 2012; 118(16):4014-4023.

24. Edlundh-Rose E, Egyházi S, Omholt K, et al. NRAS and BRAF mutations in melanoma tumours in relation to clinical characteristics: a study based on mutation screening by pyrosequencing. Melanoma Res. 2006;16(6):471-478.

25. Omholt K, Platz A, Kanter L, Ringborg U, Hansson J. NRAS and BRAF mutations arise early during melanoma pathogenesis and are preserved throughout tumor progression. Clin Cancer Res. 2003;9(17):6483-6488.

26. Vredeveld LC, Possik PA, Smit MA, et al. Abrogation of BRAFV600Einduced senescence by $\mathrm{PI} 3 \mathrm{~K}$ pathway activation contributes to melanomagenesis. Genes Dev. 2012;26(10):1055-1069.

27. Dhomen N, Reis-Filho JS, da Rocha Dias S, et al. Oncogenic BRAF induces melanocyte senescence and melanoma in mice. Cancer Cell. 2009;15(4):294-303.

28. Zhuang D, Mannava S, Grachtchouk V, et al. C-MYC overexpression is required for continuous suppression of oncogene-induced senescence in melanoma cells. Oncogene. 2008;27(52):6623-6634.

29. Hodis E, Watson IR, Kryukov GV, et al. A landscape of driver mutations in melanoma. Cell. 2012;150(2):251-263.

30. Moreau S, Saiag P, Aegerter P, et al. Prognostic value of BRAF (V600) mutations in melanoma patients after resection of metastatic lymph nodes. Ann Surg Oncol. Epub July 7, 2012.

31. Brissy S, Gaudy-Marqueste C, Mallet S, et al. BRAF mutation as a pejorative marker in metastatic melanoma [abstract]. J Clin Oncol. 2012;30(15 Suppl):Abstract 8555.

32. Rutkowski P, Jurkowska M, Gos A, et al. Correlations of molecular alterations in clinical stage III cutaneous melanoma with clinicalpathological features and patients outcome [abstract]. J Clin Oncol. 2012;30(15 Suppl):Abstract 8548.

33. Si L, Kong Y, Xu X, et al. Prevalence of BRAF V600E mutation in Chinese melanoma patients: large scale analysis of BRAF and NRAS mutations in a 432-case cohort. Eur J Cancer. 2012;48(1):94-100. 
34. Menzies AM, Haydu LE, Visintin L, et al. Distinguishing clinicopathologic features of patients with V600E and V600K BRAF-mutant metastatic melanoma. Clin Cancer Res. 2012;18(12):3242-3249.

35. Arkenau HT, Kefford R, Long GV. Targeting BRAF for patients with melanoma. Br J Cancer. 2011;104(3):392-398.

36. Ugurel S, Thirumaran RK, Bloethner S, et al. B-RAF and N-RAS mutations are preserved during short time in vitro propagation and differentially impact prognosis. PLoS One. 2007;2(2):e236.

37. Cheng S, Chu P, Hinshaw M, Smith K, Maize J, Sferruzza A. Frequency of mutations associated with targeted therapy in malignant melanoma patients [abstract]. J Clin Oncol. 2011;29(15 Suppl):S549, Abstract 8597.

38. Amanuel B, Grieu F, Kular J, Millward M, Iacopetta B. Incidence of BRAF p.Val600Glu and p.Val600Lys mutations in a consecutive series of 183 metastatic melanoma patients from a high incidence region. Pathology. 2012;44(4):357-359.

39. Bauer J, Büttner P, Murali R, et al. BRAF mutations in cutaneous melanoma are independently associated with age, anatomic site of the primary tumor, and the degree of solar elastosis at the primary tumor site. Pigment Cell Melanoma Res. 2011;24(2):345-351.

40. Maldonado JL, Fridlyand J, Patel H, et al. Determinants of BRAF mutations in primary melanomas. J Natl Cancer Inst. 2003;95(24): 1878-1890.

41. Rimoldi D, Salvi S, Liénard D, et al. Lack of BRAF mutations in uveal melanoma. Cancer Res. 2003;63(18):5712-5715.

42. Van Raamsdonk CD, Bezrookove V, Green G, et al. Frequent somatic mutations of GNAQ in uveal melanoma and blue naevi. Nature. 2009;457(7229):599-602.

43. Van Raamsdonk CD, Griewank KG, Crosby MB, et al. Mutations in GNA11 in uveal melanoma. $N$ Engl J Med. 2010;363(23):2191-2199.

44. Zhang J, Yang PL, Gray NS. Targeting cancer with small molecule kinase inhibitors. Nat Rev Cancer. 2009;9(1):28-39.

45. Wilhelm SM, Carter C, Tang L, et al. BAY 43-9006 exhibits broad spectrum oral antitumor activity and targets the RAF/MEK/ERK pathway and receptor tyrosine kinases involved in tumor progression and angiogenesis. Cancer Res. 2004;64(19):7099-7109.

46. Eisen T, Ahmad T, Flaherty KT, et al. Sorafenib in advanced melanoma: a phase II randomised discontinuation trial analysis. $\mathrm{Br} J$ Cancer. 2006;95(5):581-586.

47. Flaherty KT, Redlinger M, Schuchter LM, Lathia CD, Weber BL, O'Dwyer PJ. Phase I/II, pharmacokinetic and pharmacodynamic trial of BAY 43-9006 alone in patients with metastatic melanoma [abstract]. J Clin Oncol. 2005;23(16 Suppl):Abstract 3037.

48. Flaherty KT, Schiller J, Schuchter LM, et al. A phase I trial of the oral, multikinase inhibitor sorafenib in combination with carboplatin and paclitaxel. Clin Cancer Res. 2008;14(15):4836-4842.

49. Ott PA, Hamilton A, Min C, et al. A phase II trial of sorafenib in metastatic melanoma with tissue correlates. PLoS One. 2010;5(12):e15588.

50. Stuart DD, Aardalen KM, Lorenzana EG, et al. Characterization of a novel RAF kinase inhibitor that causes target dependent tumor regression in human melanoma xenografts expressing mutant B-RAF [abstract 4856]. Proceedings of the 97th Annual Meeting of the American Association for Cancer Research; April 5, 2006; Washington, DC. Philadelphia, PA: American Association for Cancer Research; 2006.

51. Su Y, Vilgelm AE, Kelley MC, et al. RAF265 inhibits the growth of advanced human melanoma tumors. Clin Cancer Res. 2012;18(8): 2184-2198.

52. Sharfman WH, Hodi FS, Lawrence DP, et al. Results from the firstin-human (FIH) phase I study of the oral RAF inhibitor RAF265 administered daily to patients with advanced cutaneous melanoma [abstract]. J Clin Oncol. 2011;29(15 Suppl):Abstract 8508.

53. Bollag G, Hirth P, Tsai J, et al. Clinical efficacy of a RAF inhibitor needs broad target blockade in BRAF-mutant melanoma. Nature. 2010;467(7315):596-599.

54. Falchook GS, Long GV, Kurzrock R, et al. Dabrafenib in patients with melanoma, untreated brain metastases, and other solid tumours: a phase 1 dose-escalation trial. Lancet. 2012;379(9829):1893-1901.
55. Anforth R, Fernandez-Peñas P, Long GV. Cutaneous toxicities of RAF inhibitors. Lancet Oncol. In press 2012.

56. Halait H, Demartin K, Shah S, et al. Analytical performance of a real-time PCR-based assay for V600 mutations in the BRAF gene, used as the companion diagnostic test for the novel BRAF inhibitor vemurafenib in metastatic melanoma. Diagn Mol Pathol. 2012; 21(1):1-8.

57. Anderson S, Bloom KJ, Vallera DU, et al. Multisite analytic performance studies of a real-time polymerase chain reaction assay for the detection of BRAF V600E mutations in formalin-fixed, paraffinembedded tissue specimens of malignant melanoma. Arch Pathol Lab Med. 2012;136(11):1385-1391.

58. Qu KZ, Pan Q, Zhang X, et al. Detection of BRAF mutations in melanoma: rate of mutation detection at codon 600 using Sanger sequencing as compared to the cobas 4800 method [abstract]. J Clin Oncol. 2012;30(15 Suppl):Abstract 8596.

59. Martin-Algarra S, Labiano T, Echeveste JI, et al. Use of cobas 4800 BRAF mutation test for the analysis of BRAF V600 mutations in cytological samples (CS) from metastatic melanoma (MM) [abstract]. $J$ Clin Oncol. 2012;30(15 Suppl):Abstract 8572.

60. Sosman JA, Kim KB, Schuchter L, et al. Survival in BRAF V600mutant advanced melanoma treated with vemurafenib. $N$ Engl J Med. 2012;366(8):707-714.

61. Chapman PB, Hauschild A, Robert C, et al; BRIM-3 Study Group. Improved survival with vemurafenib in melanoma with BRAF V600E mutation. N Engl J Med. 2011;364(26):2507-2516.

62. Chapman PB, Hauschild A, Robert C, et al. Updated overall survival (OS) results for BRIM-3, a phase III randomized, open-label, multicenter trial comparing BRAF inhibitor vemurafenib (vem) with dacarbazine (DTIC) in previously untreated patients with BRAFV600E-mutated melanoma [abstract]. J Clin Oncol. 2012; 30(15 Suppl):Abstract 8502 .

63. Eisenhauer EA, Therasse P, Bogaerts J, et al. New response evaluation criteria in solid tumours: revised RECIST guideline (version 1.1). Eur J Cancer. 2009;45(2):228-247.

64. Dummer R, Goldinger SM, Turtschi CP, et al. Open-label pilot study of vemurafenib in previously treated metastatic melanoma $(\mathrm{mM})$ patients (pts) with symptomatic brain metastases (BM) [abstract]. Ann Oncol. 2012;23:ix366, Abstract 1125P.

65. Laquerre S, Arnone M, Moss K, et al. A selective RAF kinase inhibitor induces cell death and tumor regression of human cancer cell lines encoding B-RAFV600E mutation [abstract]. Mol Cancer Ther. 2009;8(12 Suppl 1):Abstract B88.

66. Kefford R, Arkenau H, Brown MP, et al. Phase I/II study of GSK2118436, a selective inhibitor of oncogenic mutant BRAF kinase, in patients with metastatic melanoma and other solid tumors [abstract]. J Clin Oncol. 2010:28(15 Suppl):S611, Abstract 8503.

67. Kefford R, Arkenau HT, Brown M, et al. Selective inhibition of oncogenic BRAF V600E/K/D by GSK2118436: evidence of clinical activity in subjects with metastatic melanoma [abstract]. Pigment Cell Melanoma Res. 2010;23(6):912, Abstract 929.

68. Therasse P, Arbuck SG, Eisenhauer EA, et al. New guidelines to evaluate the response to treatment in solid tumors: European Organization for Research and Treatment of Cancer, National Cancer Institute of the United States, National Cancer Institute of Canada. J Natl Cancer Inst. 2000;92(3):205-216.

69. Trefzer U, Minor DR, Ribas A, et al. BREAK-2: a phase IIA trial of the selective BRAF kinase inhibitor GSK2118436 in patients with BRAF (V600E/K)-positive metastatic melanoma [abstract]. Pigment Cell Melanoma Res. 2011;24:1020, Abstract LBA1021-1021.

70. Long GV, Trefzer U, Davies MA, et al. Dabrafenib in patients with Val600Glu or Val600Lys BRAF-mutant melanoma metastatic to the brain (BREAK-MB): a multicentre, open-label, phase 2 study. Lancet Oncol. 2012;13(11):1087-1095.

71. Gounder MM, Spriggs DR. Inclusion of patients with brain metastases in phase I trials: an unmet need. Clin Cancer Res. 2011;17(12): 3855-3857. 
72. Raizer JJ, Hwu WJ, Panageas KS, et al. Brain and leptomeningeal metastases from cutaneous melanoma: survival outcomes based on clinical features. Neuro Oncol. 2008;10(2):199-207.

73. Azer MWF, Menzies AM, Haydu L, Kefford R, Long GV. Patterns of progression in patients (pts) with V600 BRAF-mutated melanoma metastatic to the brain treated with dabrafenib (GSK2118436) [abstract]. J Clin Oncol. 2012;30(15 Suppl):Abstract 8558.

74. Agarwala SS, Kirkwood JM, Gore M, et al. Temozolomide for the treatment of brain metastases associated with metastatic melanoma: a phase II study. J Clin Oncol. 2004;22(11):2101-2107.

75. Davies MA, Liu P, McIntyre S, et al. Prognostic factors for survival in melanoma patients with brain metastases. Cancer. 2011;117(8):1687-1696.

76. Carlino MS, Fogarty GB, Long GV. Treatment of melanoma brain metastases: a new paradigm. Cancer J. 2012;18(2):208-212.

77. Poulikakos PI, Zhang C, Bollag G, Shokat KM, Rosen N. RAF inhibitors transactivate RAF dimers and ERK signalling in cells with wild-type BRAF. Nature. 2010;464(7287):427-430.

78. Heidorn SJ, Milagre C, Whittaker S, et al. Kinase-dead BRAF and oncogenic RAS cooperate to drive tumor progression through CRAF. Cell. 2010;140(2):209-221.

79. Hatzivassiliou G, Song K, Yen I, et al. RAF inhibitors prime wild-type RAF to activate the MAPK pathway and enhance growth. Nature. 2010;464(7287):431-435.

80. Lacouture ME, Chapman PB, Ribas A, et al. Presence of frequent underlying $R A S$ mutations in cutaneous squamous cell carcinomas and keratoacanthomas (cuSCC/KA) that develop in patients during vemurafenib therapy [abstract]. J Clin Oncol. 2011;29(15 Suppl):Abstract 8520.

81. Anforth R, Tembe V, Blumetti T, Fernandez-Peñas P. Mutational analysis of cutaneous squamous cell carcinomas and verrucal keratosis in patients taking BRAF inhibitors. Pigment Cell Melanoma Res. 2012;25(5):569-572.

82. Su F, Viros A, Milagre C, et al. RAS mutations in cutaneous squamouscell carcinomas in patients treated with BRAF inhibitors. NEngl JMed. 2012;366(3):207-215.

83. Oberholzer PA, Kee D, Dziunycz P, et al. RAS mutations are associated with the development of cutaneous squamous cell tumors in patients treated with RAF inhibitors. J Clin Oncol. 2012;30(3):316-321.

84. Carlino MS, Saunders CA, Haydu LE, et al. (18)F-labelled fluorodeoxyglucose-positron emission tomography (FDG-PET) heterogeneity of response is prognostic in dabrafenib treated BRAF mutant metastatic melanoma. Eur J Cancer. Epub September 13, 2012.

85. McArthur GA, Puzanov I, Amaravadi R, et al. Marked, homogeneous, and early $[18 \mathrm{~F}]$ fluorodeoxyglucose-positron emission tomography responses to vemurafenib in BRAF-mutant advanced melanoma. J Clin Oncol. 2012;30(14):1628-1634.

86. Wilmott JS, Tembe V, Howle JR, et al. Intratumoral molecular heterogeneity in a BRAF-mutant, BRAF inhibitor-resistant melanoma: a case illustrating the challenges for personalized medicine. Mol Cancer Ther. Epub September 7, 2012.

87. Rebecca VW, Smalley KS. Tumor heterogeneity and strategies to overcome kinase inhibitor resistance in cancer: lessons from melanoma. Expert Opin Investig Drugs. 2011;20(2):137-140.

88. Wilmott JS, Long GV, Howle JR, et al. Selective BRAF inhibitors induce marked T-cell infiltration into human metastatic melanoma. Clin Cancer Res. 2012;18(5):1386-1394.

89. Sosman JA, Pavlick AC, Schuchter LM, et al. Analysis of molecular mechanisms of response and resistance to vemurafenib (vem) in BRAFV600E melanoma [abstract]. J Clin Oncol. 2012;30(15 Suppl):Abstract 8503.

90. McArthur GA, Ribas A, Chapman PB, et al. Molecular analyses from a phase I trial of vemurafenib to study mechanism of action (MOA) and resistance in repeated biopsies from BRAF mutation-positive metastatic melanoma patients (pts) [abstract]. J Clin Oncol. 2011; 29(15 Suppl):Abstract 8502.

91. Nathanson KL, Martin A, Letrero R, et al. Tumor genetic analyses of patients with metastatic melanoma treated with the BRAF inhibitor GSK2118436 (GSK436) [abstract]. J Clin Oncol. 2011; 29(15 Suppl):Abstract 8501.
92. Smalley KS, Lioni M, Dalla Palma M, et al. Increased cyclin D1 expression can mediate BRAF inhibitor resistance in BRAF V600Emutated melanomas. Mol Cancer Ther. 2008;7(9):2876-2883.

93. Straussman R, Morikawa T, Shee K, et al. Tumour micro-environment elicits innate resistance to RAF inhibitors through HGF secretion. Nature. 2012;487(7408):500-504.

94. Shi H, Moriceau G, Kong X, et al. Melanoma whole-exome sequencing identifies (V600E)B-RAF amplification-mediated acquired B-RAF inhibitor resistance. Nature Commun. 2012;3:724.

95. Poulikakos PI, Persaud Y, Janakiraman M, et al. RAF inhibitor resistance is mediated by dimerization of aberrantly spliced BRAF(V600E). Nature. 2011;480(7377):387-390.

96. Villanueva J, Vultur A, Lee JT, et al. Acquired resistance to BRAF inhibitors mediated by a RAF kinase switch in melanoma can be overcome by cotargeting MEK and IGF-1R/PI3K. Cancer Cell. 2010;18(6):683-695.

97. Montagut C, Sharma SV, Shioda T, et al. Elevated CRAF as a potential mechanism of acquired resistance to BRAF inhibition in melanoma. Cancer Res. 2008;68(12):4853-4861.

98. Nazarian R, Shi H, Wang Q, et al. Melanomas acquire resistance to B-RAF(V600E) inhibition by RTK or N-RAS upregulation. Nature. 2010;468(7326):973-977.

99. Wagle N, Emery C, Berger MF, et al. Dissecting therapeutic resistance to RAF inhibition in melanoma by tumor genomic profiling. $J$ Clin Oncol. 2011;29(22):3085-3096.

100. Johannessen CM, Boehm JS, Kim SY, et al. COT drives resistance to RAF inhibition through MAP kinase pathway reactivation. Nature. 2010;468(7326):968-972.

101. Gramza AW, Corless CL, Heinrich MC. Resistance to tyrosine kinase inhibitors in gastrointestinal stromal tumors. Clin Cancer Res. 2009;15(24):7510-7518.

102. Nguyen KS, Kobayashi S, Costa DB. Acquired resistance to epidermal growth factor receptor tyrosine kinase inhibitors in non-small-cell lung cancers dependent on the epidermal growth factor receptor pathway. Clin Lung Cancer. 2009;10(4):281-289.

103. Falchook GS, Lewis KD, Infante JR, et al. Activity of the oral MEK inhibitor trametinib in patients with advanced melanoma: a phase 1 dose-escalation trial. Lancet Oncol. 2012;13(8):782-789.

104. Kim KB, Lewis KD, Pavlick AC, et al. A phase II study of the MEK1/MEK2 inhibitor GSK1120212 in metastatic BRAF-V600E or K mutant cutaneous melanoma patients previously treated with or without a BRAF inhibitor [abstract]. Pigment Cell Melanoma Res. 2011;24:1021, Abstract LBA1021-1023.

105. Flaherty KT, Robert C, Hersey P, et al; METRIC Study Group. Improved survival with MEK inhibition in BRAF-mutated melanoma. N Engl J Med. 2012;367(2):107-114.

106. Flaherty KT, Infante JR, Daud A, et al. Combined BRAF and MEK inhibition in melanoma with BRAF V600 mutations. $N$ Engl J Med. 2012;367(18):1694-1703.

107. Flaherty K, Infante JR, Falchook GS, et al. Phase I/II expansion cohort of BRAF inhibitor GSK2118436 + MEK inhibitor GSK1120212 in patients with BRAF mutant metastatic melanoma who progressed on a prior BRAF inhibitor [abstract]. Pigment Cell Melanoma Res. 2011;24:1022, Abstract LBA1021-1024.

108. Lee CI, Menzies AM, Haydu L, Clements A, Kefford R, Long GV. Correlates of fever in patients (pts) receiving combined dabrafenib (GSK2118436) plus trametinib (GSK1120212) for V600 BRAFmutant metastatic melanoma (MM) [abstract]. J Clin Oncol. 2012; 30(15 Suppl):Abstract e19011.

109. Robert C, Thomas L, Bondarenko I, et al. Ipilimumab plus dacarbazine for previously untreated metastatic melanoma. $N$ Engl J Med. 2011; 364(26):2517-2526.

110. Hodi FS, O'Day SJ, McDermott DF, et al. Improved survival with ipilimumab in patients with metastatic melanoma. $N$ Engl $\mathrm{J} \mathrm{Med}$. 2010;363(8):711-723

111. Margolin K, Ernstoff MS, Hamid O, et al. Ipilimumab in patients with melanoma and brain metastases: an open-label, phase 2 trial. Lancet Oncol. 2012;13(5):459-465. 
112. Patnaik A, Kang SP, Tolcher AW, et al. Phase I study of MK-3475 (anti-PD-1 monoclonal antibody) in patients with advanced solid tumors [abstract]. J Clin Oncol. 2012;30(15 Suppl):Abstract 2512.

113. Topalian SL, Hodi FS, Brahmer JR, et al. Safety, activity, and immune correlates of anti-PD-1 antibody in cancer. $N$ Engl J Med. 2012;366(26):2443-2454.

114. Brahmer JR, Tykodi SS, Chow LQ, et al. Safety and activity of antiPD-L1 antibody in patients with advanced cancer. $N$ Engl J Med. 2012;366(26):2455-2465.

115. Boni A, Cogdill AP, Dang P, et al. Selective BRAFV600E inhibition enhances T-cell recognition of melanoma without affecting lymphocyte function. Cancer Res. 2010;70(13):5213-5219.

116. Mocellin S, Pasquali S, Rossi CR, Nitti D. Interferon alpha adjuvant therapy in patients with high-risk melanoma: a systematic review and meta-analysis. J Natl Cancer Inst. 2010;102(7):493-501.

117. Petrella T, Verma S, Spithoff K, Quirt I, McCready D; Melanoma Disease Site Group. Adjuvant interferon therapy for patients at high risk for recurrent melanoma: an updated systematic review and practice guideline. Clin Oncol (R Coll Radiol). 2012;24(6):413-423.
118. Hoeflich KP, Herter S, Tien J, et al. Antitumor efficacy of the novel RAF inhibitor GDC-0879 is predicted by BRAFV600E mutational status and sustained extracellular signal-regulated kinase/ mitogen-activated protein kinase pathway suppression. Cancer Res. 2009;69(7):3042-3051.

119. Torti VR, Wojciechowicz D, Hu W, et al. Epithelial tissue hyperplasia induced by the RAF inhibitor PF-04880594 is attenuated by a clinically well-tolerated dose of the MEK inhibitor PD-0325901. Mol Cancer Ther. 2012;11(10):2274-2283.

120. Schwartz GK, Robertson S, Shen A, et al. A phase I study of XL281, a selective oral RAF kinase inhibitor, in patients with advanced solid tumors [abstract]. J Clin Oncol. 2009;27(15 Suppl):Abstract 3513.

121. Ribas A, Flaherty KT. BRAF targeted therapy changes the treatment paradigm in melanoma. Nat Rev Clin Oncol. 2011;8(7):426-433.

122. Hauschild A, Grob JJ, Demidov LV, et al. Phase III, randomized, open-label, multicenter trial (BREAK-3) comparing the BRAF kinase inhibitor dabrafenib (GSK2118436) with dacarbazine (DTIC) in patients with BRAFV600E-mutated melanoma [abstract]. J Clin Oncol. 2012;30(15 Supp1):Abstract LBA8500.

\section{Publish your work in this journal}

Drug Design, Development and Therapy is an international, peerreviewed open-access journal that spans the spectrum of drug design and development through to clinical applications. Clinical outcomes, patient safety, and programs for the development and effective, safe, and sustained use of medicines are a feature of the journal, which

\section{Dovepress}

has also been accepted for indexing on PubMed Central. The manuscript management system is completely online and includes a very quick and fair peer-review system, which is all easy to use. Visit http://www.dovepress.com/testimonials.php to read real quotes from published authors.

Submit your manuscript here: http://www.dovepress.com/drug-design-development-and-therapy-journal 